DRAFT VERSION MARCH 21, 2022

Preprint typeset using LTEX style emulateapj v. 5/2/11

\title{
FERROMAGNETISM AND PARTICLE COLLISIONS: APPLICATIONS TO PROTOPLANETARY DISKS AND THE METEORITICAL RECORD
}

\author{
ALEXANDER HUBBARD ${ }^{1}$ \\ (Dated: March 21, 2022, Revision: 1.20 ) \\ Draft version March 21, 2022
}

\begin{abstract}
The meteoritical record shows both iron partitioning and tungsten isotopic partitioning between matrix and chondrules. Tungsten is not abundant enough to have driven its own isotopic partitioning, but if tungsten were correlated with iron, then ferromagnetic interactions grains could help explain both observations. We derive a practical parameterization for the increase in particle-particle collision rates caused by mutually attracting particle magnetic dipole moments. While the appropriate magnetic parameters remain uncertain, we show that ambient magnetic fields in protoplanetary disks are expected to be strong enough to magnetize iron metal bearing dust grains sufficiently to drive large increases in their collision rates. Such increased collision rates between iron metal rich grains could help preserve primordial iron and W isotopic inhomogeneities; and would help explain why the meteoritical record shows their partitioning in the solar nebula. The importance of magnetic interactions for larger grains whose growth is balanced by fragmentation is less clear, and will require future laboratory or numerical studies.

Subject headings: meteorites — protoplanetary disks — magnetic fields — planets and satellites: formation — planets and satellites: composition
\end{abstract}

\section{INTRODUCTION}

Laboratory examinations of meteorites provide a fascinating window into dust processing during the first stages of planet formation. Chondritic meteorites, being undifferentiated, record the nebular state of the dust with some fidelity (Lodders 2003). In particular, they are named after the chondrules they contain: sub-mm melted glassy beads (Hewins 1997). While chondrules were clearly dramatically thermally processed, they nonetheless inform us about their collisionally grown precursors.

One bizarre feature of chondrites is that chondrules are strongly depleted of iron relative to the inter-chondrule, nonthermally processed matrix material. Indeed, iron metal abundance is one of the primary vectors along which meteoritical compositions vary (Grossman \& Wasson 1982). There have been many studies of the behavior of iron under chondrule forming temperatures, and the difficulty in reproducing observations argues strongly for differing chondrules to have been formed in regions of differing chemical abundances (Grossman 1996, Cohen \& Hewins 2004, Ebel et al. 2008). While large variations in rare element abundances might be expected through nugget effects, iron represents approximately $20 \%$ of a chondrite's mass (Lodders 2003). Chondrules precursors would have been formed by agglomerating billions of extremely sticky (Güttler et al.2010) sub-micron pre-solar dust grains, and that agglomeration process should have washed out any primordial compositional inhomogeneities. Nonetheless, to reproduce observations it seems necessary for iron, or some other element abundant enough to react with a significant fraction of the iron, to have been partitioned between different classes of dust grain. It is unclear how that could have occurred.

Budde et al. (2015) further found tungsten isotopic partitioning between chondrules and matrix. Tungsten is extremely refractory and isotopic differences do not alter chem-

ahubbardeamnh. org

${ }^{1}$ American Museum of Natural History, New York, NY, USA istry, making the $\mathrm{W}$ isotopic partitioning even more puzzling than that of iron. Tungsten is also far too rare to affect the process of dust growth itself. The observed W isotope partitioning requires that tungsten have been carried into the solar nebula by at least two different families of pre-solar grains with different isotopic abundances. It further requires that the dust coagulation process have kept those families separate, and finally that the chondrule formation process have treated those families differently.

One way for iron, and tungsten isotopes, to have been partitioned between matrix and chondrules is for matrix and chondrules to have been produced in different spatial locations, or at different times, from ambient dust of different pre-solar origins. This possibility is unlikely because of complementarity: the composition of matrix and chondrules are strongly correlated (Hezel \& Palme 2010). Further, Goldberg et al. (2015) showed that that correlation requires matrix and chondrules to have been co-genetic. Accordingly, there is a need for a model of dust growth which takes a well-mixed initial cloud of dust and manages to preserve primordial inhomogeneities between grains within that cloud during the dust coagulation phase. The model must further allow for the chondrule formation to treat dust grains of differing compositions differently.

\section{INVOKING MAGNETIC INTERACTIONS}

In this paper, we mostly leave aside the chondrule formation process, which we discuss in a companion paper (Hubbard 2016). Instead, we focus on the dust coagulation. We require a process for preserving dust families with differing primordial compositions that can operate even at the sub-micron interstellar grain scale (Draine 2003). Given that tiny interstellar grains are extremely sticky (Güttler et al. 2010), this rules out processes that only effect the surface stickiness: the need to preserve the families starting from interstellar, sub-micron, completely sticky grains means that the composition of the grains needs to correlate with the collision rate itself, not just the sticking fraction rate of the collisions. We will also address the issue of how large the difference in the dust coagu- 
lation process needs to be for the families to end up with different aerodynamics. Differing dust aerodynamics allows for dust spatial sorting, which would allow a spatially restricted chondrule formation mechanism to process the families at different rates as is discussed in detail in Hubbard (2016).

In Hubbard (2014) we examined how dust-dust collisions were affected by the interaction of magnetic dipole moments induced in their iron-metal component by the strong nebular magnetic fields expected in the solar nebula near Mercury's orbit. We found that the magnetic interactions were extremely strong, and could erode silicates leaving behind large iron metal rich dust particles. Inspired by that work, and by the iron partitioning problem, we here examine how important it is to include the interaction of magnetic dipoles induced by the much weaker nebular magnetic fields expected in protoplanetary disks at the orbital position of the asteroid belt. Given the strong uncertainties in many of the parameters, in particular the abundance and relative magnetic permeability of astrophysical iron-nickel alloys, our primary result is the derivation a practical parameterization for how effectively magnetic dipole interactions promote dust grain collisions and collisional growth.

That parameterization suggests that magnetic interactions should indeed have played a major role in the first stages of dust growth, dramatically enhancing the dust-dust collision rates and promoting collisions between iron-rich dust grains over collisions between iron-poor grains. That latter would have helped preserve primordial iron inhomogeneities. It also provides a way to preserve primordial tungsten isotopic anomalies, so long as those anomalies were correlated with grain ferro-magnetism. However the magnitude of the effect is much lower at the dust size scale where collisional growth is balanced by fragmentation. We suggest that while magnetic attraction would not have strongly altered that equilibrium grain size, it could have helped prevent iron metal rich dust grain sub-components from fragmenting, allowing magnetic erosion (Hubbard 2014) to have proceeded, generating iron metal inhomogeneities. Further, as is discussed in Hubbard (2016), even modest differences in equilibrium grain size could lead to significant differences in chondrule processing rates.

\section{INTERACTIONS BETWEEN MAGNETIZED DUST GRAINS}

\subsection{Parameter definitions}

We follow the formalism of Hubbard (2014), with $m, m_{i}$ and $f \equiv m_{i} / m$ denoting a dust grain's total mass, iron metal mass, and metal mass fraction respectively. Dust grains are assumed spherical, with radii $a$, density $\rho$ and volume

$$
\forall=\frac{4 \pi}{3} a^{3}=\frac{m}{\rho},
$$

of which

$$
\forall_{i}=\frac{m_{i}}{\rho_{i}}
$$

is taken up by metal with density $\rho_{i}$. These definitions allow us to calculate the metal volume fraction $g$ :

$$
g \equiv \frac{\forall_{i}}{\forall}=\frac{m_{i} \rho}{m \rho_{i}}=f \frac{\rho}{\rho_{i}} .
$$

Note that collisionally aggregated dust grains in protoplanetary disks are expected to be porous. Accordingly, the densi-
Table 1

Magnetic interaction parameters

\begin{tabular}{lll}
\hline Symbol & Normalization & Parameter \\
\hline$\mu_{r}$ & 1000 & Metal relative permeability \\
$f$ & 0.2 & Metal mass fraction \\
$g$ & 0.0076 & Metal volume fraction \\
$\phi$ & 0.1 & Grain volume filling fraction \\
$\beta$ & 100 & Plasma beta \\
$\rho_{g}$ & $10^{-11} \mathrm{~g} \mathrm{~cm}^{-3}$ & Gas density (MMSN at 2.5 AU) \\
$\Sigma_{g}$ & $430 \mathrm{~g} \mathrm{~cm}^{-3}$ & Gas surface density (MMSN at 2.5 AU) \\
$T$ & $177 \mathrm{~K}$ & Gas temperature (MMSN at 2.5 AU) \\
$c_{s}$ & $8 \times 10^{4} \mathrm{~cm} \mathrm{~s}^{-1}$ & Gas sound speed (MMSN at 2.5 AU) \\
$\bar{m}$ & $2.33 \mathrm{amu}$ & Gas mean molecular mass \\
$\bar{C}$ & 0.2 & Cross-section parameter (Equation 25 \\
\hline
\end{tabular}

ties of iron metal, fused solids, and porous solids, are, respectively (Friedrich et al.|2014):

$$
\begin{aligned}
& \rho_{i} \simeq 7.86 \mathrm{~g} \mathrm{~cm}^{-3}, \\
& \rho_{s} \simeq 3 \mathrm{~g} \mathrm{~cm}^{-3}, \\
& \rho=\phi \rho_{s},
\end{aligned}
$$

where $\phi$ is the volume filling fraction,

Outside of limited regions of extremely strong magnetic fields (Hubbard 2014), the ambient magnetic field $H$ in protoplanetary disks is generally too weak to magnetize iron metal near to saturation 2 We therefore use the relationship

$$
M=\frac{\mu_{r} H}{4 \pi},
$$

where $M$ is the magnetization induced by the ambient field and $\mu_{r}$ is the magnetic relative permeability when considering magnetically soft materials, which are the main thrust of this paper. Further, we assume that the iron metal is evenly distributed throughout our dust grains. We parameterize the ambient magnetic field $H$ in terms of the thermal pressure through the plasma $\beta$ parameter:

$$
\beta \equiv \frac{8 \pi n k_{B} T}{H^{2}}=\frac{8 \pi \rho_{g} k_{B} T}{\bar{m} H^{2}},
$$

where $n$ is the number density of the gas, $\rho_{g}$ its density and $\bar{m}$ its the mean molecular mass. It follows that

$$
H^{2}=\frac{8 \pi \rho_{g} k_{B} T}{\bar{m} \beta} .
$$

Given the strongly radially decreasing gas density expected in protoplanetary disks, Equation (9) implies that there should exist an outer radius outside of which induced magnetization is negligible.

\subsection{Parameter normalizations}

We use the gas parameters of a Hayashi MMSN's midplane at $R=2.5 \mathrm{AU}$, appropriate for the asteroid belt (Hayashi 1981). Unfortunately, most of the magnetic parameters used in this paper are poorly constrained. The values we normalize to, denoted with overbars, are listed in Table 1 . The relative permeability of iron metal, $\mu_{r}$, depends on its manufacture, composition, history and the amplitude of the ambient field, potentially varying from 0 up to tens of thousands for some

2 Studies of magnetic fields in protoplanetary disks generally use the $B$ field rather than the $H$ field because the contribution of magnetized solids is negligible. 
nickel-iron alloys (Weast 1975). We normalize, possibly optimistically, to $\bar{\mu}_{r}=1000$, and hope that this work will inspire future research to shed light on this parameter.

Similarly, the amplitude of the ambient magnetic field $H$ is unclear, in no small part because we do not fully understand the behavior of the field in magnetically "dead" zones such as the midplane at $R=2.5 \mathrm{AU}$ (Gammie 1996). However, recent work has suggested that the Hall effect can allow significant field growth even there (Bai 2014), implying that the term "dead zone" may be a misnomer, leading us to normalize to $\bar{\beta}=100$. For the parameters in Table 1 , and using Equations (7) and (9), we find

$$
\bar{M} \simeq 10 \mathrm{emu} / \mathrm{cc}^{3},
$$

significantly less than the saturated magnetization of iron of (Weast 1975):

$$
M_{S} \simeq 1720 \mathrm{emu} / \mathrm{cc}^{3} .
$$

The appropriate iron metal fraction for dust in protoplanetary disks is unknown, but inspired by the low iron fraction of astrophysical silicates (Draine 2003) we adopt $\bar{f} \sim 0.2$, the overall chondritic iron mass fraction (Lodders 2003). To estimate the parameter $g$ we also need an estimate for the overall grain density (or equivalently the overall grain volume filling factor). Collisionally grown grains are expected to be highly porous, but as the collisional speeds approach ones capable of rearranging the grains, the grains will be collisionally compacted and the volume filling fractions will rise (Seizinger et al. 2012). We adopt $\bar{\phi}=0.1$, which when inserted into Equations (3) through (6) implies that

$$
\bar{g}=\bar{f} \frac{\bar{\phi} \rho_{s}}{\rho_{i}}=0.0076,
$$

although we will be invoking grains both enriched in metallic iron (higher $f, g$ ), and depleted in metallic iron (lower $f, g$ ).

\subsection{Magnetically enhanced collisional cross section: magnetically soft case}

Consider two dust grains 1 and 2 close to each other, and embedded in an external magnetic field. It is easiest to operate in either the limit of either identically sized grains or asymptotically different sized grains with $a_{1} \gg a_{2}$. The theory of turbulently stirred dust collisions is far simpler in the latter case, so we will henceforth require $a_{1} \gg a_{2}$. Accordingly, we place our larger grain 1 at the origin and consider it fixed, while following the motion of the smaller grain 2 . We will assume that the dust grains are perfectly magnetically soft: i.e. that their magnetizations are controlled purely by a spatially uniform external magnetic field $H$ according to Equation (7); and we adopt a spherical coordinate system with $\theta$ the polar coordinate and the axis aligned with the external field (and hence the magnetic dipole moments). For simplicity we assume that non-size dependent parameters other than $f$ and $g$, i.e. $\rho, \phi, \mu_{r}$, and $M$, are identical for both grains.

The magnetic energy of the dipole-dipole interaction is

$$
U_{M}=-\frac{1}{r^{3}}\left[3\left(M_{1} \cdot \hat{r}\right)\left(M_{2} \cdot \hat{r}\right)-M_{1} \cdot M_{2}\right],
$$

where $\hat{r}$ is the unit vector joining the two grains, and $M_{1,2}$ are the dipole moments. Because we have assumed that the dipole moments are aligned with the external field along the pole of our coordinate system, and we have assumed that the relative magnetic permeabilities of the grains are equal, Equation (13) reduces to

$$
U_{M}=\frac{\forall_{i 1} \forall_{i 2} M^{2}}{r^{3}}\left[1-3 \cos ^{2}(\theta)\right],
$$

where $(r, \theta)$ is the position of grain 2 , and $\theta=0$ is the pole.

We can calculate the radial magnetic force using Equation (14):

$$
\boldsymbol{F}_{M}=-\partial_{r} U_{M} \hat{r} .
$$

Two point masses cannot interact in a way which would change their angular momentum. Further, magnetic dipoles embedded in a uniform external magnetic field feel no net force, but only a torque acting to align them with the external field. Accordingly, even though $-\partial_{\theta} U_{M} \neq 0$, our system conserves its orbital angular momentum. This can be understood by noting that while Equation (14) is written in terms of $\theta$, the actual dependency in Equation (13) is on the alignment of the magnetic dipole moments; and the apparent force $-\partial_{\theta} U_{M}$ actually represents the torques the two grains exert on each other to align their dipole moments: the torque drives spin, rather than orbital angular momentum, and we are neglecting the spin of the particles. Because the particles would be torquing each-other to stay magnetically aligned, we would expect any spin they would develop to be aligned with, and extracted from, their orbital angular momentum. Accordingly, our treatment of the motion of the grains as spin-free will underestimate the effect of their magnetic interactions.

As noted above, by assuming that the magnetization of the two grains is purely controlled by the external field, we are neglecting the fact their magnetic dipole-dipole interactions will further work to align the dipole moments. Note that if the grains are close enough together, the dipole moment of one can induce a dipole moment in the second, which strongly alters the close-approach physics and further strengthens the attraction between the two particles (see e.g. Mehdizadeh et al. 2010). This simplification therefore results in a further underestimate of the magnetic attraction for the two grains.

We can estimate the magnetic enhancement to the collisional cross-section by considering polar orbits (constant azimuth). Using Equations (14) and (15), the magnetic force on grain 2 is

$$
F_{M}=\frac{3 \forall_{i 1} \forall_{i 2} M^{2}}{r^{4}}\left[1-3 \cos ^{2}(\theta)\right] .
$$

If grain 2 has an initial velocity $v_{\theta}=v_{0}$ at an initial position $\left(r_{0}, \theta_{0}\right)$, then it has angular momentum per unit mass

$$
L=r_{0} v_{0}
$$

and hence a polar velocity

$$
v_{\theta}(r)=\frac{L}{r} .
$$

We can use Equations (16) and (18) to write

$$
\begin{aligned}
& \frac{\partial^{2} r}{\partial t^{2}}=\frac{F_{M}}{m_{2}}+\frac{v_{\theta}^{2}}{r}=\frac{3 \forall_{i 1} \forall_{i 2} M^{2}}{m_{2} r^{4}}\left[1-3 \cos ^{2}(\theta)\right]+v_{0}^{2} \frac{r_{0}^{2}}{r^{3}}, \\
& \frac{\partial \theta}{\partial t}=\frac{v_{\theta}}{r}=\frac{v_{0} r_{0}}{r^{2}},
\end{aligned}
$$

which define the motion of grain 2 . 
Note that for $\theta_{0}=0$, the centrifugal and magnetic forces are balanced at a critical radius defined through

$$
r_{c}^{3} \equiv \frac{6 \forall_{i 1} \forall_{i 2} M^{2}}{m_{2} v_{0}^{2}},
$$

which also lets us define the dynamical time scale

$$
t_{c} \equiv \frac{r_{c}}{v_{0}} \text {. }
$$

Using Equations (21) and (22) we can rewrite Equations (19) and (20) as

$$
\begin{aligned}
& \frac{\partial^{2} \tilde{r}}{\partial \tilde{t}^{2}}=\frac{1-3 \cos ^{2}(\theta)}{2 \tilde{r}^{4}}+\frac{\left(r_{0} / r_{c}\right)^{2}}{\tilde{r}^{3}} \\
& \frac{\partial \theta}{\partial \tilde{t}}=\frac{\left(r_{0} / r_{c}\right)}{\tilde{r}^{2}}
\end{aligned}
$$

where $\tilde{r} \equiv r / r_{c}$ and $\tilde{t} \equiv t / t_{c}$.

We can integrate Equations (23) and $(24)$ forward in time for given values of $r_{0} / r_{c}$ and $\theta_{0}$, determining whether $r$ will hit zero (i.e. a magnetic collision). However, the limiting value of $r_{0} / r_{c}$ for which collisions occur (when one exists) for any given value of $\theta_{0}$ is complicated. Nonetheless, the only control parameter for Equations 23 and 24) is $r_{0} / r_{c}$, so the collisional cross-section for magnetic interactions between the two dust grains must scale with $r_{c}^{2}$. We can therefore write

$$
\sigma_{m}(v)=C \pi r_{c}^{2}
$$

for the magnetic collisional cross-section, where $C$ is a constant. For $\theta_{0}=0, r_{0} / r_{c}=0.49$ is the limiting value for which collisions occur, and we adopt $\bar{C}=0.2 \lesssim .0 .49^{2}$ as an approximation for $C$, noting that our analysis underestimates the strength of the magnetic interactions, and that grains only need to approach to $r=a_{1}+a_{2} \simeq a_{1}$ to collide.

This allows us to define the ratio of the magnetic collisional cross-section $\sigma_{m}$ to the geometrical cross-section $\sigma_{g} \equiv \pi a_{1}^{2}$ (recall that $a_{1} \gg a_{2}$ ):

$$
\frac{\sigma_{m}(v)}{\sigma_{g}}=C\left(\frac{r_{c}}{a_{1}}\right)^{2}=C\left[\frac{4 f_{1} f_{2} \phi \rho_{s} \mu_{r}^{2} \rho_{g} k_{B} T}{\beta \rho_{i}^{2} \bar{m} v^{2}}\right]^{2 / 3} .
$$

For velocities where $\sigma_{m}(v) \gg \sigma_{g}$, the magnetic cross-section applies, and when $\sigma_{m}(v) \ll \sigma_{g}$, the velocities are too high for magnetic forces to play a role, and the geometric crosssection applies. We approximate the intermediate regime by assuming that $\sigma=\max \left(\sigma_{m}, \sigma_{g}\right)$.

Using Equation (26) we can define the critical magnetic velocity $v_{m}$ such that $\sigma_{m}\left(v_{m}\right)=\sigma_{g}$ :

$$
v_{m}=2 C^{3 / 4} \sqrt{\frac{f_{1} f_{2} \phi \rho_{s} \rho_{g} k_{B} T}{\beta \bar{m}}} \frac{\mu_{r}}{\rho_{i}} .
$$

To calculate how much magnetic interactions change collision rates we will operate in terms of $v_{m}$, in which case Equation (26) becomes simply

$$
\frac{\sigma_{m}(v)}{\sigma_{g}}=\left(\frac{v_{m}}{v}\right)^{4 / 3}
$$

Using the parameters in Table 1 , we can write Equation 27) as

$$
v_{m} \simeq\left(\frac{C}{\bar{C}}\right)^{4 / 3} \sqrt{\frac{f_{1} f_{2} \phi \rho_{g} T \bar{\beta}}{\bar{f}^{2} \bar{\phi} \bar{\rho}_{g} \bar{T} \beta}} \frac{\mu_{r}}{\bar{\mu}_{r}} \times 0.2 \mathrm{~cm} \mathrm{~s}^{-1} .
$$

This estimate for $v_{m}$ is respectably high compared to estimates for the velocity at which dust grains bounced rather than stuck together in the solar nebula, and justifies the remainder of this paper (Güttler et al. 2010). Note that $v_{m} \propto$ $\rho_{g}^{1 / 2}$, so the strength of magnetic dipole interactions will generally decrease with orbital position.

\subsection{Magnetically enhanced collisional cross section: magnetically hard case}

In the case of magnetically hard magnetic dipoles not embedded in an external magnetic field, we can approximate that the magnetic torques act to keep the dipole moments optimally aligned. In that limit, Equation (14) simplifies to $U_{M}=-2 \forall_{i 1} \forall_{i 2} M^{2} / r^{3}$. In this case, a grain with initial velocity $v_{\theta}=v_{0}$ at $r_{0}=r_{c}$ would be on an unstable circular orbit, and the corresponding estimate for $C$ is $C=1$. This would be a reasonable approximation for dust grains each with only a single magnetic domain, and for which Equation 29, would become

$$
v_{m} \simeq 0.2\left(\frac{C}{\bar{C}}\right)^{4 / 3} \sqrt{\frac{f_{1} f_{2} \phi}{\bar{f}^{2} \bar{\phi}}} \frac{M}{\bar{M}} \frac{\mathrm{cm}}{\mathrm{s}} \simeq 171 \sqrt{\frac{f_{1} f_{2} \phi}{\bar{f}^{2} \bar{\phi}}} \times \frac{\mathrm{cm}}{\mathrm{s}},
$$

where we have used Equations (10) and (11). More extreme than Equation (29), this value for $v_{m}$ is large enough for magnetized interactions to dominate dust coagulation even at scales often associated with fragmentation (Güttler et al. 2010). Note that observations of interstellar dust imply that only a small fraction of interstellar iron could be in the form of such single-domain magnetically saturated iron-nickel dust grains however (Draine \& Lazarian 1999).

\section{EFFECT OF MAGNETIZATION ON COLLISION RATES}

\subsection{Turbulent collisional velocity distribution}

Our current picture for the collisional coagulation phase of dust growth at and below the size associated with chondrule precursors is one where macroscopic grains are stirred by both turbulence and Brownian motion. The degree of gas-dust coupling is captured by the dust Stokes number

$$
\mathrm{St} \equiv \tau \Omega,
$$

where $\tau$ is the dust drag time and $\Omega$ the local Keplerian frequency. Near the midplane of a vertically isothermal disk in vertical hydrostatic equilibrium we have

$$
\mathrm{St} \simeq \frac{\sqrt{2 \pi} a \rho}{\Sigma_{g}}
$$

where $a$ is a dust grain's radius, $\rho$ its density and $\Sigma_{g}$ the gas surface density, and in this paper, where relevant, we assume that $\mathrm{St} \ll 1$. In that limit, turbulence drives a relative velocity between dust grains which scales as (Voelk et al. 1980, Cuzzi \& Hogan 2003):

$$
v_{t} \propto \sqrt{\alpha \mathrm{St}} c_{s}
$$

where the strength of the turbulence is measured through the Shakura-Sunyaev $\alpha$ parameter (Shakura \& Sunyaev 1973). We can see from Equations (32) and (33) that larger dust grains collide more violently than smaller ones.

If the two dust grains are very different in size, as we assumed in Section 3, the turbulently driven velocity distribu- 
tion is quasi-Maxwellian (Hubbard 2013), and we have

$$
n\left(v, v_{0}\right)=n_{0}\left(\frac{v}{v_{0}}\right)^{2} e^{-v^{2} / 2 v_{0}^{2}},
$$

where $n(v)$ is the number density of targets with relative velocity $|v|$ per unit relative velocity. The velocity scale $v_{0}$ is controlled by Equation (33). We assume Equation (34) henceforth, noting that it also applies in the Brownian motion limit appropriate for very small particles extremely well coupled to the gas.

\subsection{Collision rates}

The rate at which a dust grain will collide with other grains at velocities between $v_{1}<v_{2}$ is

$$
R\left(v_{1}, v_{2}\right) \equiv \int_{v_{1}}^{v_{2}} d v n(v) \sigma(v) v,
$$

where $n(v)$ is the number density of targets with relative velocity $|v|$ per unit relative velocity, and $\sigma$ the effective collisional cross-section (which may depend on the relative velocity). In the absence of magnetic effects, we have only the geometric cross-section, i.e. $\sigma=\sigma_{g}$. Combining Equations (34) and (35) we find:

$$
\begin{aligned}
R_{g}\left(\frac{v_{1}}{v_{0}}, \frac{v_{2}}{v_{0}}\right) & =n_{0} \sigma_{g} v_{0}^{2} \int_{\frac{v_{1}}{v_{0}}}^{\frac{v_{2}}{v_{0}}} d u u^{3} e^{-u^{2} / 2}, \\
& =-n_{0} \sigma_{g} v_{0}^{2}\left[\left(u^{2}+2\right) e^{-u^{2} / 2}\right]_{v_{1} / v_{0}}^{v_{2} / v_{0}},
\end{aligned}
$$

where the subscript $g$ (geometric) is used for nonmagnetically mediated cases and we have performed the nondimensionalising substitution $u=v / v_{0}$. Accordingly, the total geometric collision rate is given by

$$
R_{g}=R_{g}(0, \infty)=2 n_{0} \sigma_{g} v_{0}^{2},
$$

where one must recall that $n_{0}$ is a number density per unit velocity. Note also that $R$ (and hence all its variants in this paper) is a function of everything that the distribution $n$ depends on. This reduces to $R$ also depending on $v_{0}$ if Equation (34) is assumed.

Magnetic interactions only matter for velocities $v<v_{m}$. Requiring $v_{2}<v_{m}$ we can therefore write the rate of magnetically mediated collisions as:

$$
\begin{aligned}
& R_{m}\left(\frac{v_{1}}{v_{0}}, \frac{v_{2}}{v_{0}}\right)=\int_{v_{1}}^{v_{2}} d v n\left(v, v_{0}\right) \sigma_{m}(v) v, \\
&=n_{0} \sigma_{g} v_{0}^{2 / 3} v_{m}^{4 / 3} \int_{\frac{v_{1}}{v_{0}}}^{\frac{v_{2}}{v_{0}}} d u u^{5 / 3} e^{-u^{2} / 2} \\
&=-n_{0} \sigma_{g} v_{0}^{2 / 3} v_{m}^{4 / 3}\left[\frac{2^{\frac{1}{3}}}{3} \Gamma\left(\frac{1}{3}, \frac{u^{2}}{2}\right)+\right. \\
&\left.u^{\frac{2}{3}} e^{-u^{2} / 2}\right]_{\frac{v_{1}}{v_{0}}}^{\frac{v_{2}}{v_{0}}},
\end{aligned}
$$

where $\Gamma$ is the incomplete Gamma function. The total increase in interactions due to magnetic effects is just

$$
\bar{R}_{m} \equiv R_{m}\left(0, v_{m} / v_{0}\right)-R_{g}\left(0, v_{m} / v_{0}\right),
$$

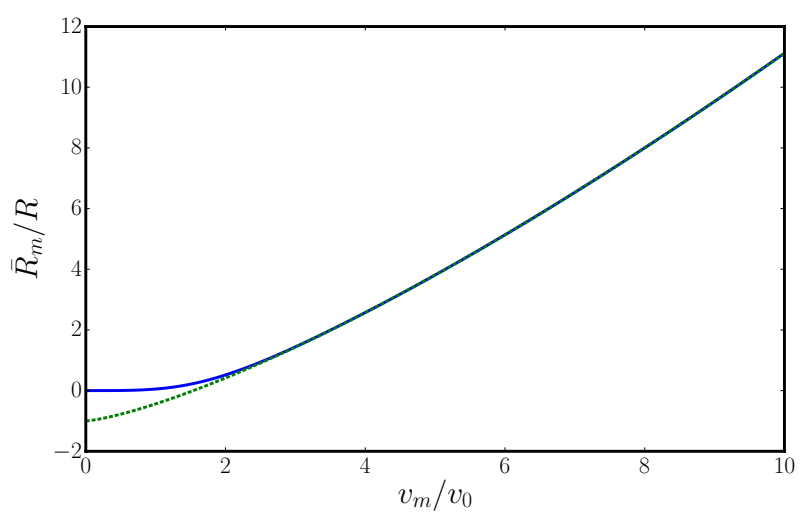

Figure 1. Blue/solid curve: fractional increase in collision rate due to magnetic interactions as a function of $v_{m} / v_{0}$ (Equation 41). Green/dashed curve: large $v_{m} / v_{0}$ approximation (Equation 42 .

and so the fractional increase is

$$
\begin{aligned}
& \frac{\bar{R}_{m}}{R_{g}}=\left(\frac{v_{m}}{v_{0}}\right)^{\frac{4}{3}}\left[\frac{2^{-\frac{2}{3}}}{3}\left[\Gamma\left(\frac{1}{3}, 0\right)-\Gamma\left(\frac{1}{3}, \frac{v_{m}^{2}}{2 v_{0}^{2}}\right)\right]\right] \\
& +e^{-v_{m}^{2} / 2 v_{0}^{2}}-1 .
\end{aligned}
$$

The important control parameter is clearly the ratio $v_{m} / v_{0}$ : if it is large, then magnetic effects are important for the bulk of the potential collisional partners, while if it is small most potential collisional partners are moving too fast for the magnetic effects to play a role.

While Equation (41) is somewhat complicated, many of its terms become negligible for large $v_{m} / v_{0}$, and we can approximate

$$
\begin{aligned}
\frac{\bar{R}_{m}}{R_{g}}\left(\frac{v_{m}}{v_{0}} \gtrsim 2\right) & \simeq \frac{2^{-\frac{2}{3}}}{3} \Gamma\left(\frac{1}{3}, 0\right)\left(\frac{v_{m}}{v_{0}}\right)^{\frac{4}{3}}-1 \\
& \simeq 0.56\left(\frac{v_{m}}{v_{0}}\right)^{\frac{4}{3}}-1 .
\end{aligned}
$$

We plot both Equations (41) and (42) in Figure 1, and one can see that the approximation is extremely good for $v_{m} / v_{0} \gtrsim 2$. Further, for $v_{m} / v_{0}>2.56, \bar{R}_{m} / R_{g}>1$ and magnetic interactions play a dominant role, more than doubling the collision rate.

\subsection{Varying $f$}

If we start with a population of dust grains with varying iron metal mass fractions, then $v_{m} \propto \sqrt{f_{1} f_{2}}$ (see Equation 27). For very small dust grains, which could even be to small to couple collisionally to turbulence and instead collide through Brownian motion (which also has a Maxwellian distribution), we expect $v_{m} \gg v_{0}$, and so $\bar{R}_{m} / R_{g} \simeq 0.56\left(v_{m} / v_{0}\right)^{4 / 3}$ (further simplifying Equation 42). In this case, the collision rate between iron rich grains is enhanced compared to that between iron poor grains, which can help preserve an initial iron inhomogeneity. Take a scenario in which half of the iron is found in metal poor grains making up $90 \%$ of the population with $f_{\mathrm{mp}}=f_{0}$ (associated with a magnetic velocity $v_{m}$ ), while the other half is found in the $10 \%$ of the grains that are metal rich with $f_{\mathrm{mr}}=9 f_{0}$. In that case, the ratio of the rates at 
which iron rich grains will collide with each other compared to colliding with iron poor grains is

$$
\frac{0.1 \times\left(f_{\mathrm{mr}}^{2}\right)^{2 / 3}}{0.9 \times\left(f_{\mathrm{mp}} f_{\mathrm{mr}}\right)^{4 / 3}} \sim 0.48
$$

over four times the ratio that would occur in the absence of magnetic interactions. While collisional growth will still act to erase inhomogeneities, magnetic interactions dramatically reduce the rate at which it does so.

\section{BOUNCING AND FRAGMENTATION}

\subsection{Bouncing barrier}

Collisional dust growth is expected to continue until the grains begin to collide rapidly enough that electrostatic sticking forces are inadequate to hold them together upon impact and they begin to bounce. This will occur for collisional velocities on the order of (Güttler et al.|2010):

$$
v_{b} \sim 10^{-2}-1 \mathrm{~cm} \mathrm{~s}^{-1} \text {. }
$$

Those estimates are mostly below that of Equation 29 , so it is interested to explore the effects of magnetic interactions on sticking rates. One must note that the release of magnetic potential energy means that magnetically mediated interactions occurring at $v=v_{b}$ will lead to collisions at velocities $v>v_{b}$. However, this velocity increase is due to the magnetic potential energy well that the collisional participants must exit for bouncing to occur; and we expect some of the kinetic energy to be thermalized or go into rearranging the dust grains. Magnetic interactions should therefore increase the effective bouncing velocity, although confirmation will require future experimental and numerical work. If the magnetically mediated collisions are violent enough, they could even lead to fragmentation, although the iron components would remain bound (e.g. magnetic erosion, Hubbard 2014). For now, we assume that the critical velocities for bouncing are not changed by consideration of magnetic effects.

Using Equation (35), we can write the sticking rate as

$$
S \equiv R\left(0, v_{b} / v_{0}\right)=\int_{0}^{v_{b}} d v n\left(v, v_{0}\right) \sigma_{\mathrm{eff}}(v) v .
$$

In the absence of magnetic interactions we can use Equation (37) to find:

$$
S_{g}=2 n_{0} \sigma_{g} v_{0}^{2}\left(1-\left[1+\frac{v_{b}^{2}}{2 v_{0}^{2}}\right] e^{-v_{b}^{2} / 2 v_{0}^{2}}\right) .
$$

Defining $v_{c} \equiv \min \left(v_{b}, v_{m}\right)$ and using Equations 39 , and 46 we can see that the increase in sticking rate due to magnetic interactions is given by

$$
\begin{gathered}
\bar{S}_{m} \equiv R_{m}\left(0, v_{c} / v_{0}\right)-R_{g}\left(0, v_{c} / v_{0}\right) \\
=n \sigma_{g} v_{0}^{2}\left(\frac{2^{\frac{1}{3}}}{3}\left(\frac{v_{m}}{v_{0}}\right)^{\frac{4}{3}}\left[\Gamma\left(\frac{1}{3}, 0\right)-\Gamma\left(\frac{1}{3}, \frac{v_{c}^{2}}{2 v_{0}^{2}}\right)\right]\right. \\
\left.+\frac{2 v_{0}^{2}+v_{c}^{2}-v_{m}^{\frac{4}{3}} v_{c}^{\frac{2}{3}}}{v_{0}^{2}} e^{-v_{c}^{2} / 2 v_{0}^{2}}-2\right)
\end{gathered}
$$

and the fractional increase is $\bar{S}_{m} / S_{g}$. We plot $\bar{S}_{m} / S_{g}$ in Figure 2. Note that for large $v_{b} / v_{0} \gtrsim 3$ the ratio is approximately independent of $v_{b}$ : almost all interactions occur for velocities $v \sim v_{0} \ll v_{b}$, so including bouncing has little effect. Note

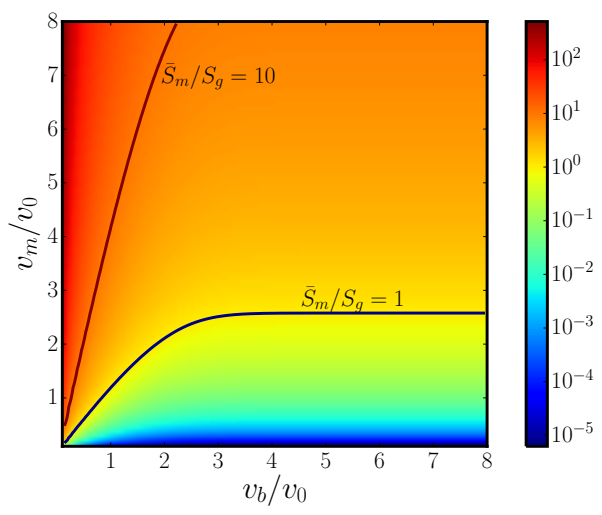

Figure 2. Fractional increase in sticking rate due to magnetic interactions as a function of $v_{m} / v_{0}$ and $v_{b} / v_{0}$. Top contour: magnetic interactions increase the sticking rate by a factor of 11 . Bottom contour: magnetic interactions increase the sticking rate by a factor of 2 .

also that even for small $v_{m} / v_{0}$, magnetic interactions can become arbitrarily important if $v_{m}>v_{b}$ : all the interactions that don't bounce are magnetically mediated.

\subsubsection{Limiting approximations}

While the full form of $\bar{S}_{m} / S_{g}$ is lengthy, one is generally in the limit that $v_{c} \gg v_{0}$ or $v_{c} \ll v_{0}$, and both limits admit simplifying approximations. In the limit that $v_{c} \gg v_{0}$, we have (using Equation 42):

$$
\frac{\bar{S}_{m}}{S_{g}} \simeq \frac{\bar{R}_{m}}{R} \simeq 0.56\left(\frac{v_{m}}{v_{0}}\right)^{\frac{4}{3}}-1 .
$$

This occurs because only a negligible fraction of encounters are at a high enough velocity for bouncing to matter.

In the limit of $v_{c} \ll v_{0}$, we can approximate Equation (34) as

$$
n \simeq n_{0}\left(\frac{v}{v_{0}}\right)^{2},
$$

leading to

$$
\begin{aligned}
& S_{g} \simeq \int_{0}^{v_{b}} d v n_{0} \sigma_{g}\left(\frac{v}{v_{0}}\right)^{2} v=n_{0} \sigma_{g} v_{0}^{2} \times \frac{1}{4}\left(\frac{v_{b}}{v_{0}}\right)^{4}, \\
& R_{g}\left(0, v_{c} / v_{0}\right) \simeq n_{0} \sigma_{g} v_{0}^{2} \times \frac{1}{4}\left(\frac{v_{c}}{v_{0}}\right)^{4} .
\end{aligned}
$$

We also have

$$
\begin{aligned}
R_{m}\left(0, v_{c} / v_{0}\right) & \simeq \int_{0}^{v_{c}} d v n_{0} \sigma_{m}\left(\frac{v}{v_{0}}\right)^{2} v \\
& \simeq n_{0} \sigma_{g} v_{0}^{2} \times \frac{3}{8}\left(\frac{v_{c}}{v_{0}}\right)^{8 / 3}\left(\frac{v_{m}}{v_{0}}\right)^{4 / 3},
\end{aligned}
$$

and hence

$$
\frac{\bar{S}_{m}}{S_{g}} \simeq \frac{3}{2} \frac{v_{c}^{8 / 3} v_{m}^{4 / 3}}{v_{b}^{4}}-\left(\frac{v_{c}}{v_{b}}\right)^{4} .
$$

We plot the ratio of these approximations for $\bar{S}_{m} / S_{q}$ to the actual value in Figure 3. We can see that Equation (49) is a good approximation as long as we have both $v_{m} / v_{0}>2.5$ and 

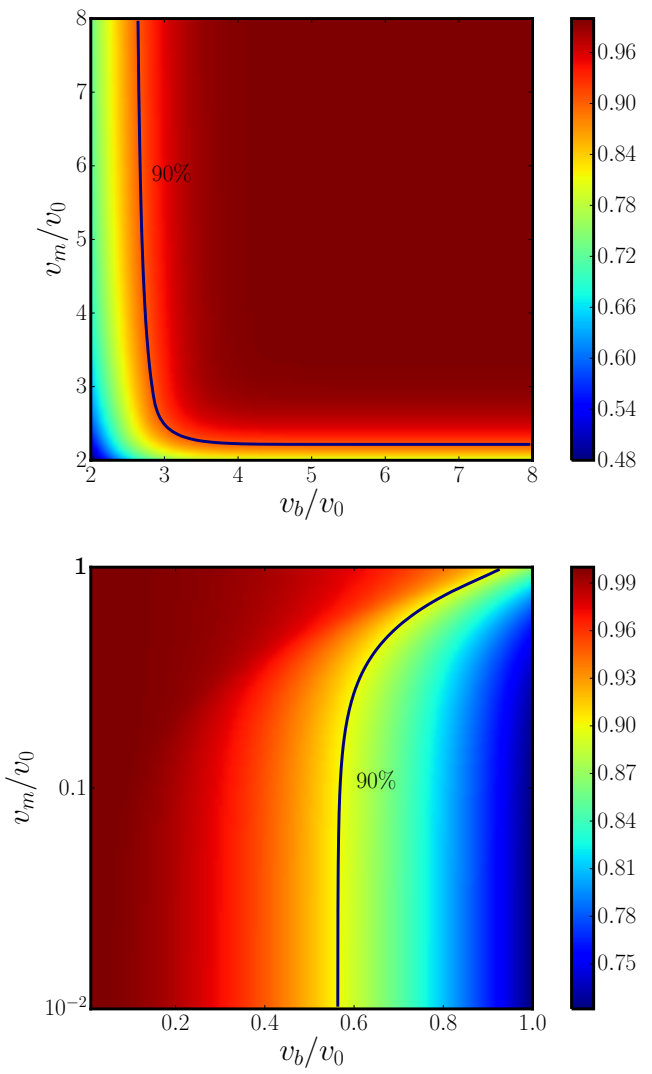

Figure 3. Top panel: Ratio of Equation (49, the large $v_{c}$ approximation for $S_{m} / S_{g}$, to the actual value. Bottom panel: Ratio of Equation [54, the small $v_{c}$ approximation for $\bar{S}_{m} / S_{g}$, to the actual value. Contours are $90 \%$.

$v_{b} / v_{0}>3$; and that Equation 54 is a good approximation for $v_{b} / v_{0}<0.5$.

\subsection{Balancing sticking and fragmentation}

In addition to bouncing, dust grains in protoplanetary disks colliding at velocities

$$
v>v_{f} \simeq 100 \mathrm{~cm} \mathrm{~s}^{-1}
$$

will fragment (Güttler et al. 2010). This value for $v_{f}$ lies well above our estimate for $v_{m}$ for magnetically soft grains (Equations 29), but is comparable to the limiting value for saturated magnetically hard grains (Equation 30). Including magnetic forces could change this picture by allowing magnetic erosion (Hubbard 2014), but exploring that in detail is far beyond the scope of this paper; and will require future experimental or numerical studies of high velocity encounters between aggregates of monomers, some of which are magnetized. Further, mass transfer is a possibility when colliding dust grains are very different in size, allowing for growth even at high collision speeds (Windmark et al.2012). For simplicity, we nonetheless adopt the picture of Equation (55) for both geometric and magnetized cases. Recall also that the collisional velocity scale $v_{0}$ is controlled by the dynamics of the disk. We have assumed turbulent stirring, with $v_{0}$ scaling with the square root of the Stokes numbers of the dust grains (see Equations 32 and 33.

At some critical collisional velocity scale, dust grain collisions that result in sticking will balance those that result in fragmentation. We parameterize this balance through

$$
R\left(0, v_{b} / v_{0}\right)=\psi R\left(v_{f} / v_{0}, \infty\right),
$$

where $\psi$ is the critical growth-neutral ratio of the sticking rate to the fragmentation rate. We also choose to define the ratio of the fragmentation and bouncing velocities as

$$
\xi \equiv v_{f} / v_{b}
$$

Note that $v_{b}, v_{f}$ and $\xi$ depend on the microphysics of the collisions, and we have assumed that they are independent of any magnetic interactions. Combining Equations (29), (44) and (55), we expect $v_{f} \gg v_{m}>v_{b}$ for magnetically soft grains. Accordingly, for a given dust grain size (and hence collisional velocity scale $v_{0}$ ), including magnetic interactions would not effect the fragmentation rate, but would increase the sticking rate. If $\psi$ is constant, then magnetized grains would find a balance between sticking and fragmentation for larger collisional velocity scales, and hence at larger dust grain sizes. As is explored in Hubbard (2016), differences in dust grain sizes leads to aerodynamical sorting which could be correlated with the chondrule formation process. Such a correlation would provide an explanation for the observed partitioning of iron, and of tungsten isotopes, between chondrules and matrix (Grossman \& Wasson 1982, Budde et al. 2015).

In the geometric case we can consider Equation (56) to be an equation for $v_{b} / v_{0}$ (and hence $v_{f} / v_{0}$ ) as a function of $\psi$ and $\xi$. In this case Equation (56) becomes

$$
2-\left[\left(\frac{v_{b}}{v_{0}}\right)^{2}+2\right] e^{-\frac{v_{b}^{2}}{2 v_{0}^{2}}}=\psi\left[\left(\frac{\xi v_{b}}{v_{0}}\right)^{2}+2\right] e^{-\frac{\xi^{2} v_{b}^{2}}{2 v_{0}^{2}}}
$$

noting that $n_{0}$ and $\sigma$ have cancelled, and $v_{0}$ only shows up in ratio with $v_{b}$. We write $u_{g}(\psi, \xi)$ as the solution of Equation 58 for $v_{b} / v_{0}$. However, it is crucial to note that we have assumed that $v_{b}$ is set by the microphysics, so $u_{g}$ actually measures $v_{0}$. Accordingly, we define

$$
v_{0, g}\left(\psi, \xi, v_{b}\right) \equiv v_{b} / u_{g},
$$

the collisional velocity scale for Equation (34) at which sticking and fragmentation are balanced for a set of parameters $\left(\psi, \xi, v_{b}\right)$.

For the magnetic case we can similarly use

$$
R_{m}\left(0, v_{b} / v_{0}\right)=\psi R_{m}\left(v_{f} / v_{0}, \infty\right)
$$

to define $u_{m}\left(\psi, \xi, v_{b}, v_{m}\right)$ as the solution of Equation 60 for $v_{b} / v_{0}$. We will use $u_{m}$ to define

$$
v_{0, m}\left(\psi, \xi, v_{b}, v_{m}\right) \equiv v_{b} / u_{m}
$$

The ratio $v_{0, m} / v_{0, g}$, which is also the ratio $u_{g} / u_{m}$ because $v_{b}$ and $v_{f}$ are not altered by magnetic interactions, describes how much more violently magnetized dust grains have to be stirred for their collisions to be growth neutral; and hence how much larger they can grow.

\subsubsection{Exploring the consequences of magnetization on the balance between sticking and fragmentation}

The ratio $v_{0, m} / v_{0, g}$ depends on too many parameters to fully explore in this paper, but by restricting some of the parameters we can estimate the impact that magnetization has on the dust grain size at which sticking and fragmentation are balanced. Based on Equations (29), 44) and (55) we adopt

$$
v_{b}=0.1 \mathrm{~cm} \mathrm{~s}^{-1}<v_{m} \ll v_{f}=100 \mathrm{~cm} \mathrm{~s}^{-1},
$$



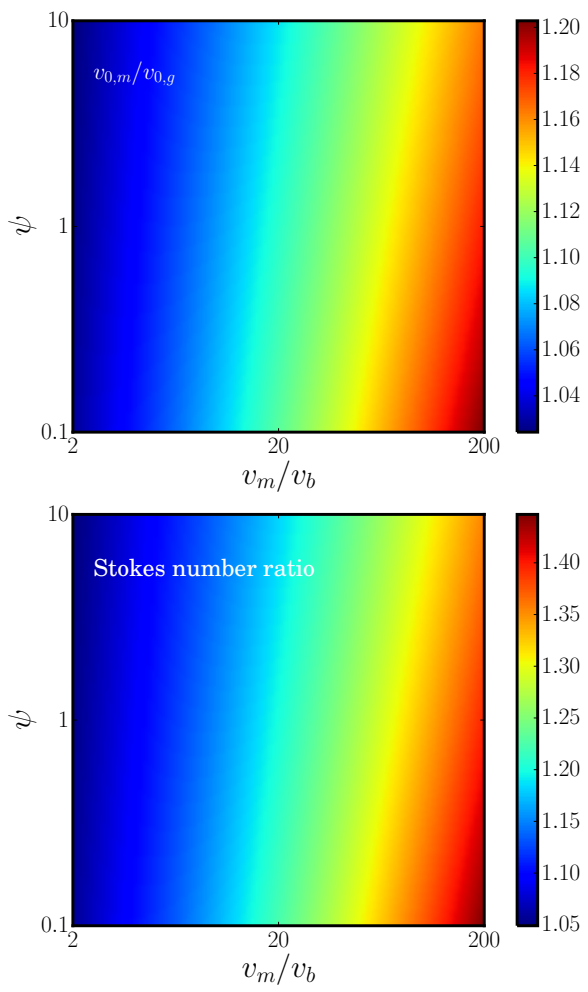

Figure 4. Top panel: Ratio of the turbulent collisional velocity scales required to balance sticking and fragmentation in the magnetized and nonmagnetized cases (the non-magnetized case does not depend on $v_{m} / v_{b}$ ). In this figure we hold $\xi \equiv v_{f} / v_{b}=1000$ fixed, varying the magnetic velocity $v_{m}$ and the growth-neutral sticking rate to fragmentation rate ratio $\psi$. Bottom panel: Ratio of the Stokes numbers of magnetically interacting growthneutral grains to those of non-magnetically interacting growth-neutral grains implied by top panel using Equation 33

i.e. $\xi=10^{3}$. Given this ratio between $v_{b}$ and $v_{f}$ we can be certain that at least one of the limits $v_{b} \ll v_{0}$ or $v_{f} \gg$ $v_{0}$ holds, and accordingly that at least one of the sticking or fragmentation rates is small. We will also assume that $\psi$, the growth-neutral ratio of the sticking to fragmentation rates is neither very large nor very small. Combining Equation 62 with this condition on $\psi$ implies that both the sticking and fragmentation rates must be small, and we have

$$
v_{b} \ll v_{0} \ll v_{f},
$$

which allows significant simplifications.

Because $v_{b} \ll v_{0}$ we can use Equation (51) to rewrite Equation (58) as

$$
4 \psi\left(\frac{\xi^{2}}{u_{g}^{2}}+\frac{2}{u_{g}^{4}}\right) e^{-\xi^{2} u_{g}^{2} / 2}-1=0 .
$$

We also have $v_{c} \equiv \min \left(v_{b}, v_{m}\right)=v_{b} \ll v_{0}$ and $v_{m} \ll v_{f}$, so we can use Equation (53) to rewrite Equation (60) as

$$
\frac{8}{3} \psi\left(\xi^{2} u_{m}^{-2 / 3}+2 u_{m}^{-8 / 3}\right) e^{-\xi^{2} u_{m}^{2} / 2}-\left(\frac{v_{m}}{v_{0}}\right)^{4 / 3}=0 .
$$

We can parameterize the magnetic velocity $v_{m}$ in terms of the bouncing velocity and $u_{m}$ through:

$$
\frac{v_{m}}{v_{0}}=\frac{v_{m} v_{0, m} v_{b}}{v_{0, m} v_{0} v_{b}}=\frac{v_{m}}{v_{b}} \frac{v_{0, m}}{v_{0}} u_{m} .
$$

Because Equation (65) is an equation for $v_{0, m}$ (through the intermediary $u_{m}$ ), its solution for $v_{0, m}$ is unchanged by setting $v_{0}=v_{0, m}$ in Equation (66). This reduces Equation (65) to

$$
\frac{8}{3} \psi\left(\xi^{2} u_{m}^{-\frac{2}{3}}+2 u_{m}^{-\frac{8}{3}}\right) e^{-\frac{\xi^{2} u_{m}^{2}}{2}}-\left(\frac{v_{m}}{v_{b}} u_{m}\right)^{\frac{4}{3}}=0 .
$$

For our fixed $\xi=10^{3}$, Equations (64) and (67) can be solved for $u_{g}$ and $u_{m}$ (and hence $v_{0, m} / v_{0, g}$ ) as functions of $\psi$ and $v_{m} / v_{b}$. In Figure 4 we plot the ratio $v_{0, m} / v_{0, g}$, along with the implied difference in Stokes number, for growth-neutral grains. One immediate observation is that the equilibrium size is only moderately shifted even when $v_{m} \gg v_{b}$ and magnetization strongly increases the sticking rate. We can understand this by taking the derivative of the fragmentation rate (i.e. the right hand side of Equation 58, with respect to $v_{0}$ :

$$
\partial_{v_{0}}\left(\psi\left[\left(\frac{v_{f}}{v_{0}}\right)^{2}+2\right] e^{-\frac{v_{f}^{2}}{2 v_{0}^{2}}}\right) \simeq \frac{\psi}{v_{0}}\left(\frac{v_{f}}{v_{0}}\right)^{4} e^{-\frac{v_{f}^{2}}{2 v_{0}^{2}}},
$$

where we have used $\xi v_{b}=v_{f} \gg v_{0}$. Dividing the fragmentation rate by its derivative we can estimate how sensitive it is to small changes in $v_{0}$ :

$$
\frac{\mathrm{RHS}}{\partial_{v_{0}} \mathrm{RHS}} \simeq v_{0}\left(\frac{v_{0}}{v_{f}}\right)^{2} \ll v_{0} .
$$

The exponential term in the fragmentation rate is an extremely sensitive function of velocity scale, so even significant changes in the sticking rate will be balanced by small changes in the collisional velocity scale.

\subsubsection{Saturated magnetization case}

In the limiting case of magnetically hard grains magnetized to saturation (Equation 30, we have $v_{m} \sim v_{f}$, and magnetic interaction must in some fashion alter the critical collisional velocity. We can estimate that if $v_{m} \gtrsim v_{f}$, where $v_{f}$ is the fragmentation speed in the absence of magnetic interactions, then the magnetic interactions allow grain survival up to a magnetic-fragmentation speed $v_{f m} \sim v_{m}$. In that case, we can use Equation (33) to estimate that the fragmentation limited dust grain size Stokes number scales as St $\propto v_{m}^{2} \propto f_{1} f_{2} \phi$. In this limiting case, magnetic interactions are expected to strongly alter the maximum size to which dust grains grow.

\subsubsection{Varying $f$}

As noted above, while including magnetic interactions can decrease the time it takes for grains to grow enough for sticking and fragmentation to balance, the magnetic interactions do not strongly change the grain size at which that balance occurs. Accordingly, iron rich and iron poor grains will have broadly similar sizes. Further, because we expect $v_{m} \ll v_{f}$, the magnetic energy that helps hold iron rich grains together is much smaller than the kinetic energy associated with fragmentation. Considerations of magnetic interactions are unlikely to significantly change $v_{f}$.

Collisional fragmentation does not generally completely destroy dust grains however, and it is possible that grains with 
inhomogeneously distributed iron will fragment in a fashion that preserves the iron rich portions. Indeed, the flip side to Equation (69) is that almost all fragmentation collisions occur at velocities only a hair above $v_{f}$, do not have a significant energy excess available, and must seek out the weakest links binding collisionally grown aggregates together. This could allow magnetic effects to play an outsized role in controlling the results of the fragmentation process.

If so, and given the more rapid regrowth of iron rich dust grains, this could not only delay the loss of primordial iron metal inhomogeneities, but generate inhomogeneities in an alternate version of magnetic erosion (Hubbard 2014). We emphasis though that the difference in energy scales means that such a process would suffer from a fine-tuning problem.

\section{CONCLUSIONS}

We have examined the effect that magnetic dipole-dipole interactions would have on dust-dust collisions. The strength of the interaction can be parameterized in a single velocity scale $v_{m}$, the critical velocity below which magnetic forces increase the effective collisional cross-section. Under the approximation of a Maxwellian large-separation dust-dust relative velocity distribution, appropriate for turbulently stirred particles of strongly differing sizes, or for Brownian motion, the inclusion of magnetic interactions can be reasonably simply encapsulated.

In general, collision rates are strongly enhanced if the magnetic velocity $v_{m}>v_{0}$, the scale of the Maxwellian velocity distribution. Further, if dust grain collisions lead to sticking only below a characteristic velocity $v_{b}$, magnetic interactions strongly increase the sticking rate if either $v_{m}>v_{0}$ or $v_{m}>v_{b}$ is satisfied. Finally, if collisions above a characteristic velocity $v_{f}$ lead to fragmentation, with $v_{f}>v_{m}$, then the inclusion of magnetic interactions will increase the sticking rate, but not the fragmentation rate, increasing the size of growth-neutral dust grains whose sticking and fragmentation rates are balanced. However, we find that this final effect is relatively minor due to the exquisite sensitivity of the fragmentation rate to the Maxwellian velocity scale.

While the appropriate parameters are extremely uncertain, and especially in the case of the relative magnetic permeability $\mu_{r}$ could vary by orders of magnitude, we have estimated for $v_{m}$ in the case of dust in protoplanetary disks whose iron metal component is magnetized by the ambient magnetic field. We find that magnetic interactions are expected to play a significant role in setting the dust-dust collision rates at least at and inwards of the orbital position of our asteroid belt, at least for the smaller grains. This means that magnetic interactions must be considered for the initial stages of dust coagulation, and dust chemistry, although the implications at the chondrule precursor scale are less certain Indeed, if pre-solar iron was carried into the solar nebula in the form of single magnetic domains and therefor maximally magnetized, magnetic interactions would play an overwhelming role. We hope that these results inspire laboratory studies to explore the magnetic properties of plausible pre-solar iron-nickel bearing grains. Such studies would not only provide better estimates for $\mu_{r}$, but would permit our model to be generalized beyond perfectly magnetically soft and perfectly magnetically hard dust grains.

Because $v_{m}$ depends on the iron metal fraction of the dust grains, magnetic forces will help preserve primordial iron metal inhomogeneities in the dust population of a protoplanetary disk, providing an explanation for the observed partitioning of iron between chondrules and matrix (Grossman \& Was- son 1982). If those iron metal inhomogeneities were correlated with tungsten isotopic inhomogeneities, magnetic forces could provide part of the puzzle for explaining the observations of Budde et al. (2015). Both cases also require that iron content be correlated with the chondrule formation process. We address this in a companion paper, Hubbard (2016), using a model where the weak dependency of equilibrium grain size on magnetic interactions leads to iron rich grains being less coupled. In that case, if chondrule formation events were limited to occurring at altitude, iron rich grains would be converted into chondrules at a slower rate than iron poor grains.

Note however that the energy scale of fragmentation is much larger than that of the magnetic interactions in the magnetically soft case, so a significant effect at the largest dust size scale, where growth and fragmentation are balanced, is only expected if magnetic forces help preserve iron rich portions of fragmenting dust grains. Most of the collisions above the critical fragmentation velocity $v_{f}$ are only just above that velocity though, and so are not expected to have a large energy excess. Therefore it is plausible that magnetic effects could play an outsized role in controlling the fragmentation products, justifying future studies of high velocity impact between collisional aggregrates, some of whose components are magnetized.

\section{ACKNOWLEDGEMENTS}

This research was inspired and greatly helped by conversations with Denton S. Ebel, Harold Connolly and Michael K. Weisberg. We thank the anonymous referee for pushing us to improve the sophistication of our analysis of the strength of the magnetic interactions. The research leading to these results was funded by NASA OSS grant NNX14AJ56G.

\section{REFERENCES}

Bai, X.-N. 2014, ApJ, 791, 137

Budde, G., Kleine, T., Kruijer, T. S., \& Metzler, K. 2015, in Lunar and

Planetary Inst. Technical Report, Vol. 46, Lunar and Planetary Science Conference, 2262

Cohen, B. A., \& Hewins, R. H. 2004, Geochim. Cosmochim. Acta, 68, 1677

Cuzzi, J. N., \& Hogan, R. C. 2003, Icarus, 164, 127

Draine, B. T. 2003, ARA\&A, 41, 241

Draine, B. T., \& Lazarian, A. 1999, ApJ, 512, 740

Ebel, D. S., Weisberg, M. K., Hertz, J., \& Campbell, A. J. 2008, Meteoritics and Planetary Science, 43, 1725

Friedrich, J. M., Weisberg, M. K., Ebel, D. S., Biltz, A. E., Corbett, B. M., Iotzov, I. V., Khan, W. S., \& Wolman, M. D. 2014, Chemie der Erde Geochemistry,

Gammie, C. F. 1996, ApJ, 457, 355

Goldberg, A. Z., Owen, J. E., \& Jacquet, E. 2015, MNRAS, 452, 4054

Grossman, J. N. 1996, in Chondrules and the Protoplanetary Disk, ed. R. H. Hewins, R. H. Jones, \& E. R. D. Scott, 243-253

Grossman, J. N., \& Wasson, J. T. 1982, Geochim. Cosmochim. Acta, 46, 1081

Güttler, C., Blum, J., Zsom, A., Ormel, C. W., \& Dullemond, C. P. 2010,

A\&A, 513, A56

Hayashi, C. 1981, Progress of Theoretical Physics Supplement, 70, 35

Hewins, R. H. 1997, Annual Review of Earth and Planetary Sciences, 25, 61

Hezel, D. C., \& Palme, H. 2010, Earth and Planetary Science Letters, 294, 85

Hubbard, A. 2013, MNRAS, 432, 1274

-. 2014, Icarus, 241, 329

-. 2016, ApJ, submitted

Lodders, K. 2003, ApJ, 591, 1220

Mehdizadeh, A., Mei, R., Klausner, J., \& Rahmatian, N. 2010, Acta Mechanica Sinica, 26, 921

Seizinger, A., Speith, R., \& Kley, W. 2012, A\&A, 541, A59

Shakura, N. I., \& Sunyaev, R. A. 1973, A\&A, 24, 337

Voelk, H. J., Jones, F. C., Morfill, G. E., \& Roeser, S. 1980, A\&A, 85, 316

Weast, R. C. 1975, CRC Handbook of chemistry and physics. A

ready-reference book of chemical and physical data

Windmark, F., Birnstiel, T., Ormel, C. W., \& Dullemond, C. P. 2012, A\&A 544, L16 